\title{
Correntropy-based Spectrum Sensing for Wireless Microphones in Man-made Noise Environments
}

\author{
Jongmin Lee and Jose C. Principe \\ Department of Electrical and Computer Engineering, \\ University of Florida, Gainesville, FL 32611-6200, USA \\ Email: jm.lee@ufl.edu, principe@cnel.ufl.edu
}

\begin{abstract}
Correntropy is a generalized similarity measure that is here applied to estimate the spectral density which we called the correntropy spectral density. When the Gaussian kernel is utilized in correntropy, there is a free parameter. In this paper, we propose a weighted method to reduce the concerns about the free parameter selection. The proposed method is applied for detecting the presence of narrow-band wireless microphone signals in TV white space. The simulation results show that the proposed method has higher detection ability than the conventional power spectral density method in man-made noise environments and very close to the conventional in Gaussian noise, while maintaining low false alarms in both.

Index Terms - correntropy, weighted correntropy spectral density, spectrum sensing, cognitive radio, non-Gaussian noise, wireless microphone.
\end{abstract}

\section{INTRODUCTION}

Spectrum sensing is a method to detect unoccupied spectrum of TV bands which is referred as TV white space (TVWS). Another method for identifying the TVWS is geolocation with database access. Recently the federal communication commission (FCC) finalized the ruling that eliminates the requirement that TV band devices (TVBDs) that incorporate geo-location and database access must also have spectrum sensing capability to identify TV signals and low-power auxiliary services such as wireless microphones (WMs). However, the so-called "sensing-only" TVBDs still require more reliable spectrum sensing techniques. These devices need certification from the FCC and the details of the sensing algorithms should be submitted [1]. Moreover, the FCC explains that spectrum sensing will play an important roll in the efficient management of RF spectrum in the future. It is also known that WMs are one of the obstacles for deployment of unlicensed secondary TVBDs because WMs are low power licensed secondary users that occupy narrow spectrum bands in TVWS. Therefore, the development of more robust spectrum sensing techniques for cognitive radio applications is necessary.

There are several challenging issues that we may have to consider for the robustness [2]. One of them is that signal level is significantly low at TVBDs so that sensors must detect the presence of a very weak signal in noise. Multipath fading is also an inssue. But if the signal used is a narrow-band feature, the multi-path fading can be considered as flat Rayleigh fading. Finally one of the most challenging issue is detection of WM signals in man-made non-Gaussian distributed noise. This noise tends to be narrow-band in nature and causes false alarms. The narrow-band WM sensor needs to overcome false alarms caused by man-made noise [3].

Correntropy in [4][5] extends the second-order statistics similarity into second and higher-order statistics by applying kernel methods. Non-Gaussian signals may not be characterized simply by second-order moments but require also higherorder statistics. As we will show, correlation is a special case of correntropy when the kernel used in the definition is a product kernel. However, when shift invariant kernels are preferred in the definition, a new set of properties emerge that are very useful to characterize a sum of moments of the random variable, preserving the nice properties of correlation that enable the existence of spectral representations.

One application is spectral density estimation. The Fourier transform of correntropy was introduced as a generalized spectral density [4], and named correntropy spectral density (CSD). In this paper, based on the CSD, we propose a weighted CSD (WCSD) to remove the free parameter inherited by the shift invariant kernel. This paper verifies experimentally that the proposed method is robust to detect WM signals in non-Gaussian distributed noise. The simulation results show that WCSD has better detection ability as well as low false alarms in man-made non-Gaussian noise, while the proposed method is very close to the conventional power spectral density in Gaussian noise.

The rest of this paper is organized as follows. In Section II, the modeling and signal generation of wireless microphone and man-made noise. In Section III, the correntropy-based spectral density is derived. And Section IV describes the weighted method. Simulation results are provided for validation and discussions in Section $\mathrm{V}$, and Section VI concludes this paper.

\section{Wireless Microphone Signal AND MAN-MADE NOISE MODEL}

Most of the wireless microphone (WM) signals utilize ana$\log$ frequency modulation (FM) for transmission in a narrow bandwidth, which is less than $200 \mathrm{kHz}$ but most systems utilize the full bandwidth to maximize audio quality [6]. Signal model generations are introduced in [7]. In this paper we use the silent mode, soft speaker, and loud speaker signal models whose spectral estimates are respectively illustrated in Fig. 1. These models represent very narrow, medium, and large 
TABLE I

WirelesS Microphone (WM) SignAL TYPES [7]

\begin{tabular}{ccc}
\hline Type & Tone & FM Deviation \\
\hline Silent Mode & $32 \mathrm{kHz}$ & $\pm 5 \mathrm{kHz}$ \\
Soft Speaker & $3.9 \mathrm{kHz}$ & $\pm 15 \mathrm{kHz}$ \\
Loud Speaker & $13.4 \mathrm{kHz}$ & $\pm 32.6 \mathrm{kHz}$
\end{tabular}

bandwidth signals within $200 \mathrm{kHz}$. Let $m(t)$ be the voice signal. The transmitted FM signal $s(t)$ can be generated by

$$
s(t)=A_{c} \cos \left(2 \pi f_{c} t+2 \pi \Delta f \int_{0}^{t} m(\tau) d \tau\right)
$$

where $A_{c}$ and $f_{c}$ are the amplitude and the carrier frequency respectively. The constant $\Delta f$ is the frequency deviation of an FM modulation. [7] introduces a simulation method and generation of WM signals. There are three types of signal generations as described in Table I. Those signals are considered in outdoor and indoor environments. In outdoor case, the WM signals are transmitted in a line of sight (LOS) between transmitter and receiver equipments. Thus, it can be modeled by additive noise. For the indoor case, because the distance between transmitter and receiver is short and the speed of user is assumed to be $0.6 \mathrm{~m} / \mathrm{s}$, a flat fading channel is considered and the maximum Doppler shift at a maximum carrier frequency of $806 \mathrm{MHz}$ is calculated to be less than $2 \mathrm{~Hz}$. The flat fading channel does not affect the detection performance of the WM signal significantly as shown in [8] because the estimated PSD of the signal maintains its shape in flat fading [9]. Although this method can detect the WM signals in additive white Gaussian noise (AWGN) and the flat fading channel model, in practice, the received signal at the TVBD may also be impaired by non-Gaussian noise such as man-made impulsive noise. We will evaluate the PSD and the correntropy-based methods in outdoor nonGaussian noise environments. If the noises are non-Gaussian distribution, detecting peaks of the PSD may not result in good performance in practice.

We assume that the received signal is added by man-made noise so that it is not necessarily Gaussian signal. Let $s(n)$ and $\varepsilon(n)$ are respectively the WM signal and additive noise which are sampled at a sampling rate $f_{s}$. And let $x(n)$ denote the received signal at a TVBD. Two hypothesis are considered:

$$
\begin{aligned}
& \mathcal{H}_{0}: x(n)=\varepsilon(n) \\
& \mathcal{H}_{1}: x(n)=s(n)+\varepsilon(n) .
\end{aligned}
$$

$N$ samples of the received signal are denoted as $\{x(n)\}_{n=0}^{N-1}$. And $\{\varepsilon(n)\}_{n=0}^{N-1}$ are independent and identically distributed (i.i.d.) random variables with zero mean but not assumed Gaussian distribution.

For the impulsive man-made noise generation, we use symmetric $\alpha$-stable $(S \alpha S)$ distribution with $1<\alpha<2$ and zero mean [10]. The $S \alpha S$ distribution is widely used to model impulsive noise behavior [10][11]. We can use this distribution to evaluate both Gaussian $(\alpha=2)$ and nonGaussian $(\alpha<2)$ cases. Although the expectation of higherorder statistics except for $\alpha=2$ is infinite, the finite-sample mean is still available. The characteristic function of the noise is given by

$$
\Psi_{\alpha}(u)=e^{-\gamma^{\alpha}|u|^{\alpha}}
$$

where $\gamma$ is a dispersion parameter which is similar to a standard deviation. Unlike the usual definition of signal-tonoise ratio, we use an alternative of signal-to-dispersion ratio given by

$$
S N R=\frac{P_{s}}{2 \gamma^{2}}
$$

where $P_{s}$ is the power of $s(n)$. When $\alpha=2$, the $S \alpha S$ is equivalent with Gaussian distribution with variance $2 \gamma^{2}$.

\section{Correntropy-based Spectral Density}

The conventional PSD method can be described as the square magnitude of the Fourier transform of the received signal, which is periodogram. So the estimate is associated with second-order statistics. If the distribution of the received signal with noise is Gaussian, the PSD is good enough to detect the primary users. However, if non-Gaussian such as impulsive noise, we may have to consider higher-order statistics. Base on correntropy [4], we use a generalized spectral density so that the spectral estimate of received signal can be more precise even in impulsive noise environments.

Let $X$ and $Y$ be two arbitrary real random variables. Generalized correlation between $X$ and $Y$ is defined as:

$V(X, Y)=E[\kappa(X, Y)]=\iint \kappa(x, y) p_{X Y}(x, y) d x d y$

where $E[\cdot]$ denotes the expectation over the joint space and $\kappa(\cdot, \cdot)$ is any continuous positive definite kernel.

The conventional cross-correlation is a special case when $\kappa(x, y)=x y$ is substituted in (6). Cross-correntropy is obtained when the kernel is shift invariant such as the Gaussian kernel given by

$$
\kappa(x, y)=G_{\sigma}(x-y)=\exp \left(-\frac{\|x-y\|^{2}}{2 \sigma^{2}}\right)
$$

where $\sigma$ is a kernel size which is a free parameter inherited by the kernel function.

Now we are working with an auto-correntropy function at two different time instances $t$ and $s$ of the random process $X(t)$. So (6) with a kernel size parameter $\sigma$ becomes

$$
V_{X ; \sigma}(t, s)=E\left[G_{\sigma}(x(t)-x(s))\right] .
$$

When only a finite number of data samples $\left\{\left(x_{n}, y_{n}\right)\right\}_{n=0}^{N-1}$ are available in practice, the sample estimator of the autocorrentropy is defined as

$$
\hat{V}_{\sigma}(m)=\frac{1}{N-m} \sum_{n=m}^{N-1} G_{\sigma}(x(n)-x(n-m))
$$



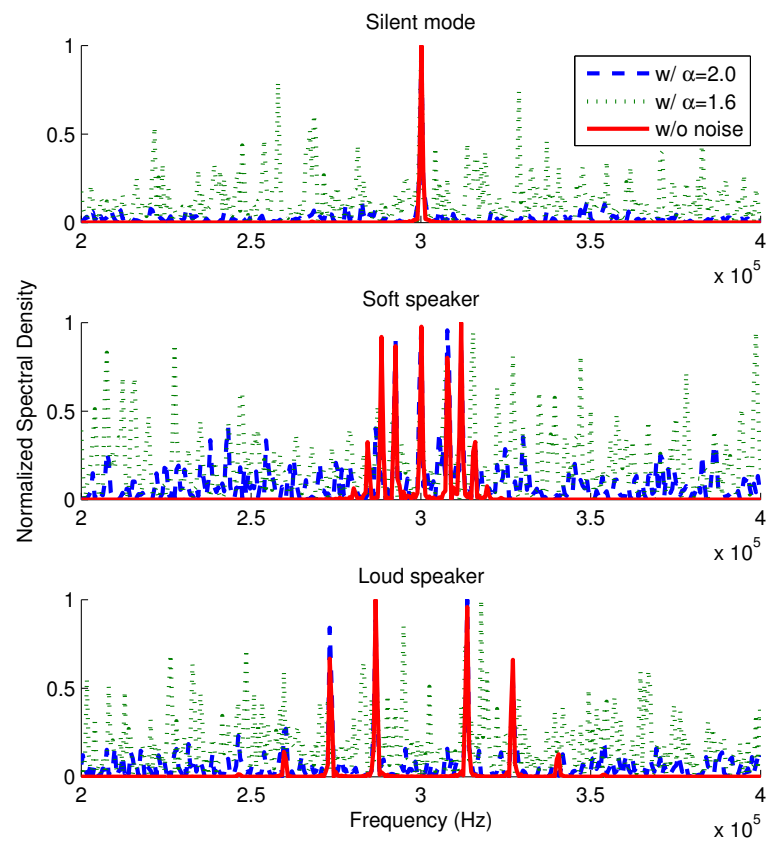

(a) Estimates of PSD
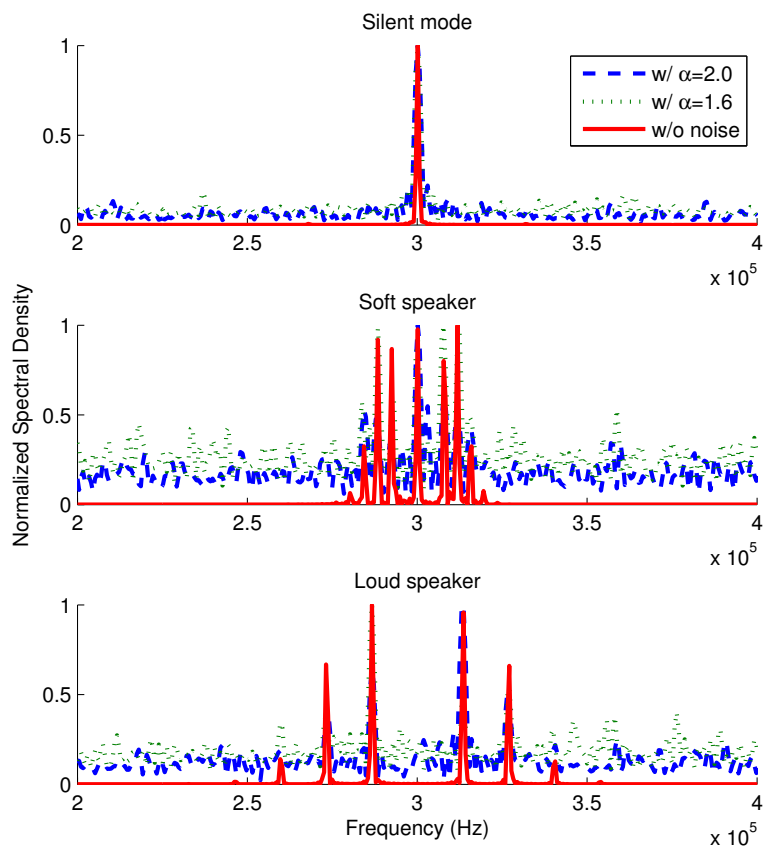

(b) Estimates of WCSD

Fig. 1. Estimates for three WM signal models (silent, soft, and loud). Gaussian $(\alpha=2)$ and non-Gaussian $(\alpha=1.6)$ noises are compared. Sensing time is $1 \mathrm{~ms}$, and $\mathrm{SNR}=-15 \mathrm{~dB}$. Spectral densities are normalized by the peak of each. The scale of $\mathrm{y}$-axis is linear.

where $X$ in $V_{X ; \sigma}(t, s)$ is dropped for simplicity and $m=t-s$.

A Taylor series expansion provides a meaningful insight of the auto-correntropy function for the Gaussian kernel. By the expansion, (8) can be described as

$$
V_{\sigma}(t, s)=\sum_{n=0}^{\infty} \frac{(-1)^{n}}{2^{n} \sigma^{2 n} n !} E\left[\|x(t)-x(s)\|^{2 n}\right]
$$

which involves all the even-order moments of the random variables $(x(t)-x(s))$. In particular, we can see that the auto-correntropy function is proportional to the conventional covariance function $R_{x}(t, s)$ when $n=1$ :

$$
\begin{array}{r}
E\left[\|x(t)\|^{2}\right]+E\left[\|x(s)\|^{2}\right]-2 E[\langle x(t), x(s)\rangle] \\
=\operatorname{var}_{x_{t}}+\operatorname{var}_{x_{s}}-2 R_{x}(t, s) .
\end{array}
$$

For $n>1$, it involves sums of all even-moments of the random variable. Moreover, when large kernel sizes are utilized, the second-order moment dominates the auto-correntropy function and it defaults to biased auto-correlation.

The kernel in the auto-correntropy function induces a scalar nonlinear mapping $\eta_{\mathbf{x}}$ that maps a random process $\{x(n)\}_{n=0}^{N-1}$ into $\left\{\eta_{x}(n)\right\}_{n=0}^{N-1}$ while preserving the similarity measure. The existence of the nonlinear mapping $\eta_{\mathbf{x}}$ for any positive definite kernel is proved in [12] and explains that positive definite kernels are covariance operators. So (8) can be rewritten with the nonlinear mapping $\eta_{\mathbf{x}}$ :

$$
\begin{aligned}
V_{\sigma}(t, s) & =E\left[G_{\sigma}(x(t)-x(s))\right] \\
& =E\left[\eta_{x}(t) \eta_{x}(s)\right] .
\end{aligned}
$$

And the generalized covariance function, which is also called the centered auto-correntropy function, defined as

$$
\begin{aligned}
U_{\sigma}(t, s)= & E_{x_{t} x_{s}}\left[G_{\sigma}(x(t)-x(s))\right] \\
& -E_{x_{t}} E_{x_{s}}\left[G_{\sigma}(x(t)-x(s))\right] .
\end{aligned}
$$

By analogy with the well-known Wiener-Kinchin theorem, the Fourier transform of the centered auto-correntropy function is named the correntropy spectral density (CSD) and is defined as

$$
S(f ; \sigma)=\sum_{m=-(L-1)}^{L-1} U(m ; \sigma) e^{-j 2 \pi f m}
$$

where $L$ is the lag size of the auto-correntropy function. The CSD is a real, even function, and nonnegative [13]. However, it is a function of kernel size $\sigma$.

\section{Weighted Correntropy Spectral Density}

Although the CSD has a big potential for spectral estimation of non-Gaussian signals, it has a free parameter - the kernel size $\sigma$ and the estimate of CSD will vary with it since the kernel size weights differently the second and higherorder moments of the random variable. Therefore, we use the weighted CSD (WCSD) to eliminate the free parameter. The baseline of the kernel size selection is obtained by Silverman's rule of thumb [14]. The kernel size has to be selected 
TABLE II

KERNEL Sizes $\sigma^{*}$ By SiLverman's Rule

(a) Silent Mode

\begin{tabular}{|c||c|c|c|c|c|}
\hline SNR (dB) & -26 & -22 & -18 & -14 & -10 \\
\hline$\alpha=2.0$ & 2.19 & 1.39 & 0.89 & 0.58 & 0.39 \\
\hline$\alpha=1.8$ & 3.44 & 2.21 & 1.38 & 0.90 & 0.59 \\
\hline$\alpha=1.6$ & 6.31 & 4.01 & 2.60 & 1.55 & 1.03 \\
\hline
\end{tabular}

(b) Soft Speaker

\begin{tabular}{|c||c|c|c|c|c|}
\hline SNR (dB) & -26 & -22 & -18 & -14 & -10 \\
\hline$\alpha=2.0$ & 2.19 & 1.39 & 0.89 & 0.58 & 0.39 \\
\hline$\alpha=1.8$ & 3.42 & 2.18 & 1.38 & 0.90 & 0.59 \\
\hline$\alpha=1.6$ & 6.32 & 4.04 & 2.54 & 1.66 & 1.04 \\
\hline
\end{tabular}

(c) Loud Speaker

\begin{tabular}{|c||c|c|c|c|c|}
\hline SNR (dB) & -26 & -22 & -18 & -14 & -10 \\
\hline$\alpha=2.0$ & 2.19 & 1.39 & 0.89 & 0.58 & 0.39 \\
\hline$\alpha=1.8$ & 3.43 & 2.18 & 1.41 & 0.90 & 0.58 \\
\hline$\alpha=1.6$ & 6.26 & 3.93 & 2.51 & 1.71 & 1.08 \\
\hline
\end{tabular}

according to data dynamics and the number of samples. The Silverman's rule for Gaussian distribution is

$$
\sigma^{*}=\sigma_{X}\left(4 N^{-1}(2 d+1)^{-1}\right)^{\frac{1}{(d+4)}},
$$

where $\sigma_{X}$ is the standard deviation of the received signal, $N$ is the number of samples, and $d$ is the data dimensionality. Although (15) is derived for Gaussian distribution we can use this rule to estimate a certain range of kernel sizes. Table II represents the kernel sizes $\sigma^{*}$ of different SNRs and $\alpha$ for the three indoor WM signal models. The values are averaged over 10,000 iterations generated random noise sequences. These values indicate that the kernel size selection depends on the received signal's power and distribution, whereas the estimated kernel size parameters are almost same among the three models.

Fig. 2 shows detection probabilities of CSD with different kernel sizes for the silent mode WM signal in Gaussian and non-Gaussian noises. We can see that there are appropriate kernel sizes to maximize the detection probability. Since the kernel size selection may result in different detection ability, we will show that the weighted method averaging over set of kernel sizes results in almost constant detection capability regardless of kernel size selection and noise distribution. Assuming that the received signal's SNR is very low (e.g., less than $-10 \mathrm{~dB}$ ) and that the impulsive behavior is defined by the parameter $1.6 \leq \alpha \leq 2.0$, we initiate simulations with the kernel sizes between 2 and 18. Different range of kernel sizes will be shown in Fig. 4.

The CSD $S(f ; \sigma)$ of (14) is a function of kernel size $\sigma$. Thus, the weighted CSD (WCSD) is defined by

$$
R(f)=\frac{1}{K} \sum_{k=0}^{K-1} S\left(f ; \sigma_{k}\right) .
$$

By substituting (14) into (16) and replacing the expectation operator with sample mean, the WCSD can be estimated as

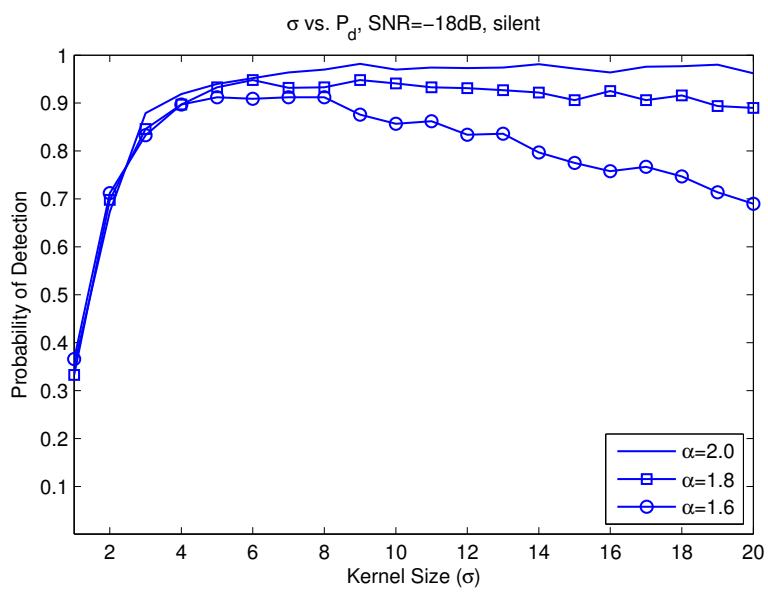

Fig. 2. Kernel sizes vs. detection probability of CSD. Silent mode signal. $\mathrm{SNR}=-18 \mathrm{~dB}$, ts $=0.8 \mathrm{~ms}$. $\alpha=2.0$ for Gaussian noise, others are for non-Gaussian.

below:

$$
\begin{aligned}
\hat{R}(f) & =\frac{1}{K} \sum_{k=0}^{K-1}\left[\sum_{m=-(L-1)}^{L-1} \hat{U}\left(m ; \sigma_{k}\right) e^{-j 2 \pi f m}\right] \\
& =\sum_{k=0}^{K-1} w(k) \sum_{m=-(L-1)}^{L-1} \hat{U}\left(m ; \sigma_{k}\right) e^{-j 2 \pi f m}
\end{aligned}
$$

Note that $w(k)$ is a weight function of kernel size index $k$. In this paper we only consider $w(k)$ as equal weights for $\sigma_{k}$. Then, the test statistic is given by

$$
T=\max _{f} \hat{R}(f) \text {. }
$$

The threshold is determined by experimental noise distribution and desired false alarm probability.

\section{Simulation Results}

In our simulations to evaluate detection capability of WCSD for narrow-band wireless microphone (WM) signals in Gaussian and non-Gaussian noises, we used the three types of models: silent mode, soft speaker, and loud speaker, described in Section II. The indoor model results in similar performance with outdoor when a sensor detects peaks of spectral density [8] because the fading channels almost look like flat fading in narrow-band. Thus, we test the indoor case to find differences between non-Gaussian and Gaussian noise effects.

If WM system utilizes $200 \mathrm{kHz}$ for a bandwidth, 30 channels in $6 \mathrm{MHz}$ TV white space are available. But sensing threshold power is averaged over $200 \mathrm{kHz}$. In our model, both the signal and noise are generated in $0.6 \mathrm{MHz}$ bandwidth with $1.2 \mathrm{MHz}$ of sampling frequency, and the WM signal is located at $300 \mathrm{kHz}$. As we can see the signal's shapes in Fig. 1 , there are narrow-band peaks within $200 \mathrm{kHz}$. That means the signal-to-noise ratio (SNR) is boosted by $\frac{6 \times 10^{5}}{2 \times 10^{5}} \approx 5 \mathrm{~dB}$ within $200 \mathrm{kHz}$, while $\frac{6 \times 10^{6}}{2 \times 10^{5}} \approx 15 \mathrm{~dB}$ for $6 \mathrm{MHz} \mathrm{TV}$ white space. Furthermore, since longer sensing time results in better 


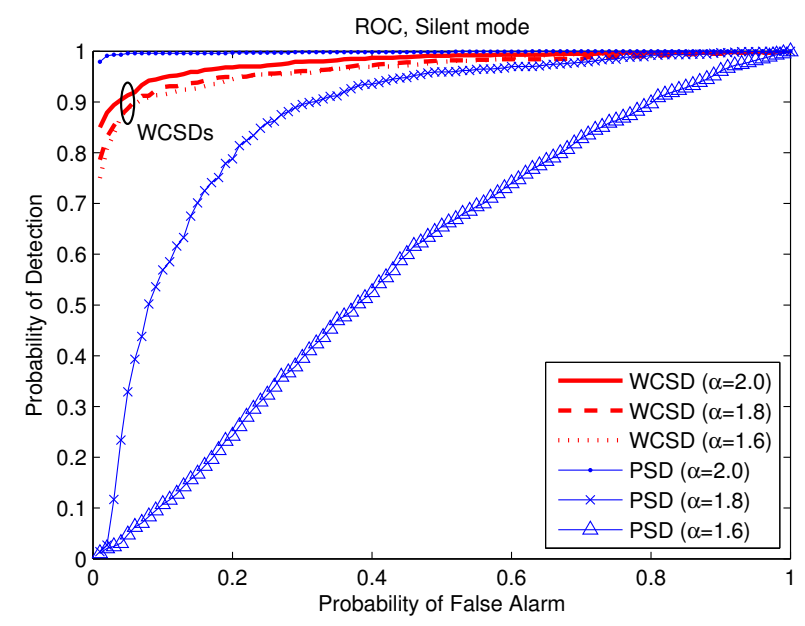

(a) Silent mode

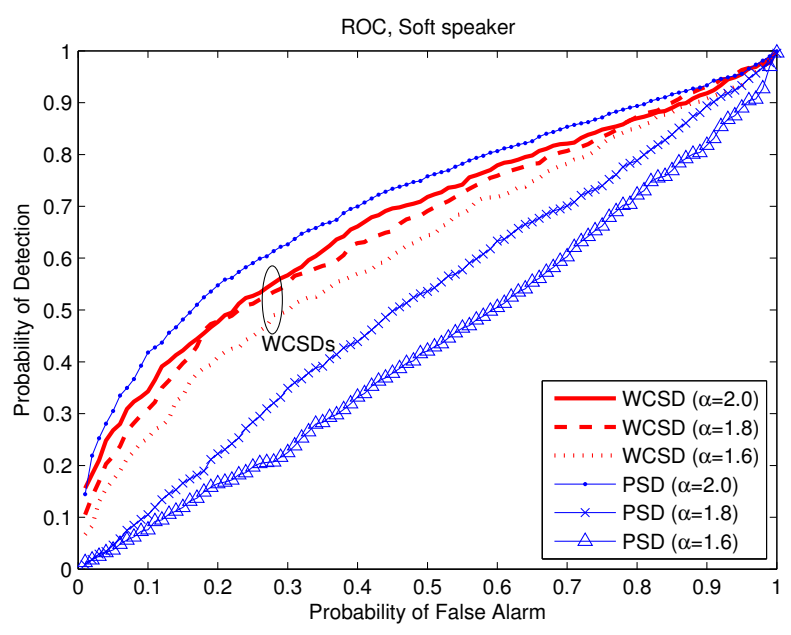

(b) Soft speaker

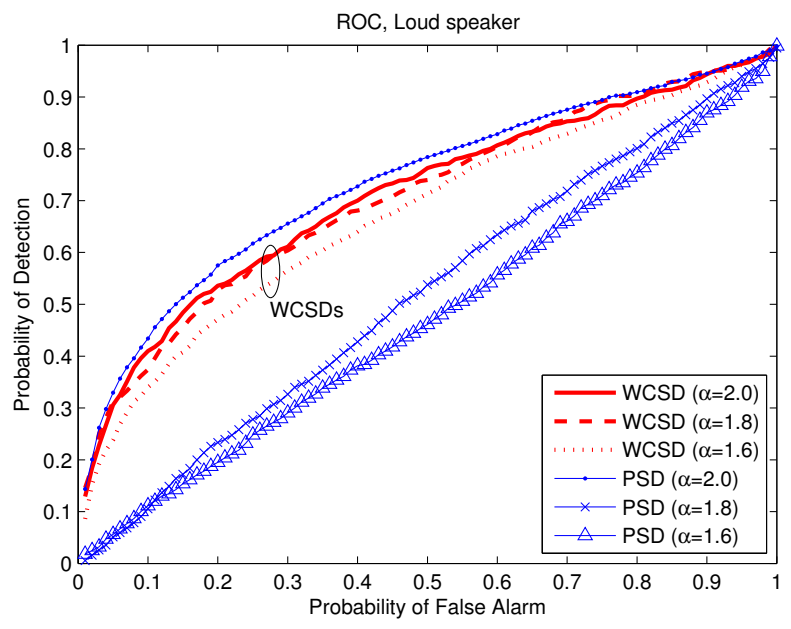

(c) Loud speaker

Fig. 3. Receiver operating characteristic (ROC) for the three models of WM. $t_{s}=0.8 \mathrm{~ms}$ and $\mathrm{SNR}=-18 \mathrm{~dB}$. detection probability, our following simulation results can be improved for larger bandwidth and longer sensing time.

Fig. 1 compares estimates of PSD with those of WCSD, in non-Gaussian $(\alpha=1.6)$ and Gaussian $(\alpha=2)$ distributed noises for the three types of WM signals. Since the PSD is based on the second-order moment, the non-Gaussian noise might be not characterized so that its estimate has a lot of spikes out of the signal's peaks. However, the WCSD mitigates those spikes by characterizing higher-order statistics as well as second-order.

Fig. 2 describes the effects of kernel size selection for CSD. As we can see, there is difference of the detection probabilities with various kernel sizes and noise distributions. If we use CSD for non-Gaussian signal's spectrum estimation, we may have to find appropriate kernel sizes that maximize sensing performance.

By WCSD, however, we can eliminate such a free parameter. Fig. 3 shows the receiver operating curves (ROCs) for the three different signal models in Gaussian and non-Gaussian noises. From those figures, we can see that the WCSD is much better than PSD in non-Gaussian noises, while very close to the PSD in Gaussian. As the impulsive behavior becomes larger (e.g., from $\alpha=2.0$ to 1.6), the sensing performance of the WCSD does not change very much while that of PSD degrades. The silent mode signal has better detection than others because the estimate has relatively a high peak in the middle of bandwidth as shown in Fig. 1.

Fig. 4 compares the ROCs between the WCSDs of various kernel size selections and the CSD of too small and too large kernel sizes. If the CSD selects too small or too large kernel sizes in non-Gaussian noise, the detection ability will degrade significantly because the weighting of the moments is not appropriate. The second-order moment becomes dominant for large $\sigma$, while higher-order moments are emphasized with a small $\sigma$. In Fig. 4(a), since the noise is non-Gaussian ( $\alpha=1.6$ ), both $\sigma=50$ and $\sigma=0.2$ degrade the detection probability. On the other hand, Fig. 4(b) shows the Gaussian noise $(\alpha=2.0)$ can be discriminated by large kernel size $\sigma=50$. However, the WCSD that averages over seven CSDs with different $\sigma$ does not change very much so that its detection probability can be made less dependent of the kernel size.

\section{CONCLUSION}

One of the most challenging issue of spectrum sensing for TVBDs is detection of narrow-band wireless microphones in man-made non-Gaussian noise because it makes the spectrum sensing difficult and causes large false alarms. We used the correntropy-based spectral density methods to detect the wireless microphones in impulsive man-made noise environments. Symmetric $\alpha$-stable distribution was used to model such impulsive noises. Since the CSD has a free parameter - kernel size, the sensing performance may vary with different kernel sizes. Therefore, we proposed the weighted CSD (WCSD) to eliminate the kernel size dependency. The range of kernel size candidates to be weighted can be estimated by Silverman's 


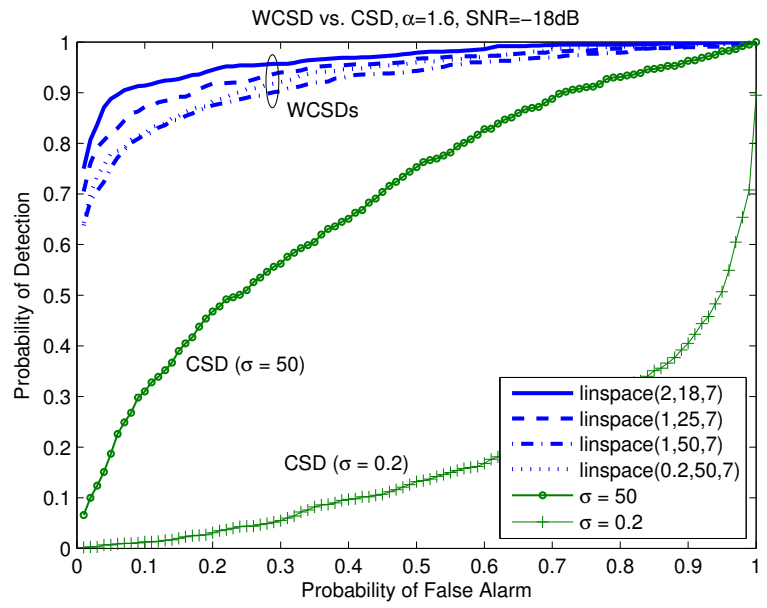

(a) $\alpha=1.6$

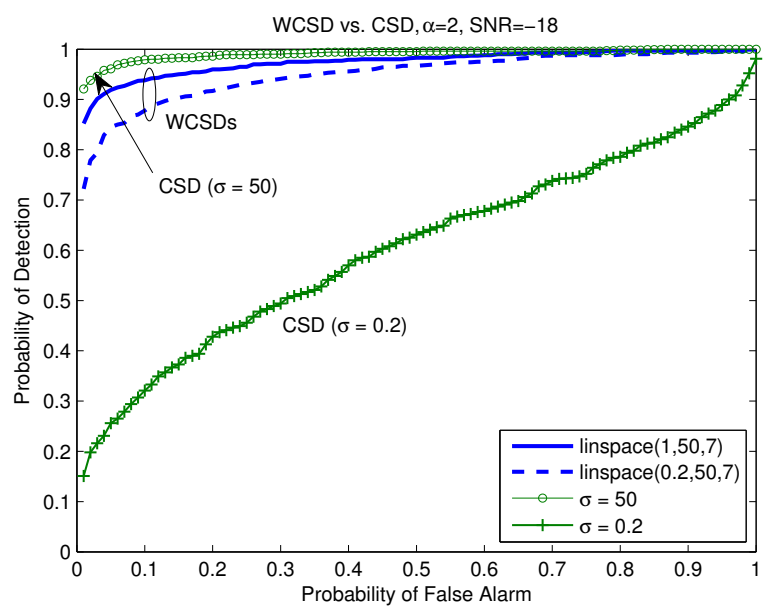

(b) $\alpha=2.0$

Fig. 4. WCSD vs. CSD with different windows of kernel sizes. For WCSD, linspace $(a, b, 7)$ denotes 7 equally separated kernel sizes from a to $b$ are selected with equal weights. Two CSDs with $\sigma=0.2$ and $\sigma=50$ are compared with the WCSDs.

rule. Three models of the wireless microphone signals was considered and the experimental results show that the proposed method has better detection ability than conventional power spectral density in non-Gaussian noise and very close to the conventional in Gaussian, while maintaining low false alarms.

\section{REFERENCES}

[1] FCC 10-174, "Second Memorandum Opinion and Order."

[2] S. J. ShellHammer, A. K. Sadek, and W. Zhang, "Technical Challenges for Cognitive Radio in TV White Space Spectrum," UCSD Information Theory and Applications Workshop, January 2009.

[3] R. Balamurthi, H. Joshi, C. Nguyen, A. K. Sadek, S. J. Shellhammer, and C. Shen, "A TV White Space Spectrum Sensing Prototype," IEEE Symposium on DySPAN, pp. 297-307, May 2011.

[4] J. C. Principe, Information Theoretic Learning: Renyi's Entropy and Kernel Perspectives, Springer, 2010.

[5] W. Liu, P. P. Pokharel, and J. C. Principe, "Correntropy: Properties and Applications in Non-Gaussian Signal processing," IEEE Trans. on Signal Processing, vol. 55, no. 11, pp. 5286-5298, 2007.

[6] E. Reihl, "Wireless Microphone Characteristics," IEEE 802.2206/0070r0, May 2006
[7] C. Clanton, M. Kenkel, and Y. Tang, "Wireless Microphone Signal Simulation Method," IEEE 802.22-07/0124r0, Mar. 2007.

[8] H. Chen, W. Gao, and D. G. Daut, "Spectrum Sensing for Wireless Microphone Signals," in Proc. of IEEE SECON Workshop pp. 1-5, June 2007.

[9] T. S. Rappaport, Wireless Communications, Principles and Practice, 2nd ed., Prentice Hall, 2002.

[10] C L. Nikias, M. Shao, Signal Processing with Alpha-stable Distributions and Applications, Wiley, New York, 1995.

[11] J. H. McCulloch, Financial applications of stable distributions, in: G. S. Madala, C. R. Rao (Eds.), Handbook of Statistics, vol. 14, Elsevier, Amsterdam, pp. 393-425, 1996.

[12] E. Parzen, "Statistical inference on time series by Hilbert space methods," Tech. Report 23, Stat. Dept., Stanford Univ., 1959.

[13] J. Xu, "Nonlinear Signal Processing Based on Reproducing Kernel Hilbert Space," Ph.D. Dissertation, University of Florida, 2008.

[14] B. Silverman, Density Estimation for Statistics and Data Analysis, Chapman and Hall, London, 1986. 